\title{
A portable pupilometer system for measuring pupillary size and light reflex
}

\author{
ALEKSANDRAS RADZIUS, PHYLLIS WELCH, EDWARD J. CONE, \\ and JACK E. HENNINGFIELD \\ Addiction Research Center, National Institute on Drug Abuse, Baltimore, Maryland
}

\begin{abstract}
A new pupilometry assessment system was evaluated as an instrument for potential use in basic research and clinical studies of addictive drug use in humans. The system is portable and can be used as a peripheral device with a variety of IBM-compatible personal computers. The pupilometer electronically measures the pupil diameter with infrared sensors and has the ability to stimulate the pupil with a programmable pulse of light and to record the resultant pupillary response. The present report compares the static pupil-diameter measurements obtained with this infrared pupilometer and those obtained photographically in a bench-top and in a clinical setting. The infrared pupilometer was also used to record the light reflex of the pupil in research subjects after administration of various morphine-related drugs. The results indicate that static pupil-diameter measurements made with the infrared pupilometer compare favorably with the photographic measurement. The ability of the infrared pupilometer to record the light reflex may provide an additional means of quantitatively measuring drug action.
\end{abstract}

Pupil diameter has been one of the basic quantitative physiological indices of exposure to psychoactive drugs (Himmelsbach, 1941; Tennant, 1988). The photographic instrument commonly used in research is a close-up camera (CU camera), the Polaroid CU-5 (Jasinski \& Martin, 1967; Marquardt, Martin, \& Jasinski, 1967). Infrared pupilometers have occasionally been used in drug studies (Henningfield, Miyasato, Johnson, \& Jasinski, 1983; Pickworth \& Lee, 1988), but their considerable expense, difficulty in use, and lack of portability have precluded widespread application.

In the present report, we describe the operation and reliability of a new instrument for measuring pupil diameters in either a static or a dynamic mode. The Fairville Medical Optics Pupilscan is is an infrared electronic pupilometer that measures, displays, and records pupil diameters and has the additional capacity to stimulate the pupil with a light pulse and record the ensuing pupillary response. ${ }^{1}$ The rates of constriction and dilation elicited by the light stimulus may provide additional parameters for studying drug-induced effects.

Two series of tests were conducted. In the first, the capabilities of the pupilometer were evaluated and compared with those of the CU camera. In the second, pupildiameter measurements made with the pupilometer were compared with those made with the CU camera, on human volunteers in a clinical setting.

The U.S. government does not endorse or favor any specific commercial product or company. Trade or company names appearing in this article are used only because they are considered essential in the context of the studies reported. We thank Mrs. Eleanor W. Waldrop for her helpful comments on the manuscript. Correspondence should be addressed to Jack E. Henningfield, NIDA Addiction Research Center, P.O. Box 5180, Baltimore, MD 21224.

\section{Description}

The pupilometer is a hand-held device that functions as peripheral hardware for IBM personal computers, including lap-top models, and IBM compatibles.

The system consists of four elements: (1) an optical unit (described below); (2) a driver circuit board designed to operate the electronics of the optical unit and generate a real-time image of the pupil to aid in positioning the optical unit accurately; (3) an auxiliary monitor to display the pupil image; and (4) an operating program supplied on a floppy disk. The driver circuit board functions as a plug-in accessory in an expansion slot in the computer. Later versions of the program display the pupil image on the host monitor, obviating the need for an auxiliary monitor.

The face of the optical unit contains a light-emitting diode as a low-angle viewing light, to aid in aligning the optical unit with the pupil. The intensity of the viewing lamp is fixed at $3.5 \mathrm{~lm} / \mathrm{ft}^{2}$. In the face of the optical unit are a pair of high-intensity green diodes used to stimulate the pupil with a pulse of light. Two pairs of infrared diodes, also in the face of the optical unit, illuminate the pupil, and the resulting reflected image is focused on an electronic image sensor (see Figure 1).

The subject's eye is viewed directly through the main tube of the optical unit, and the proper focal distance is assured with the use of a clip-on padded plastic cheekrest placed against the subject's cheekbone.

\section{Operation}

The optical unit is operated with a switch (see Figure 1). When the switch is depressed, the viewing lamp and the infrared diodes are turned on and measurement of the pupil image begins. Measures of the pupil are made ev- 


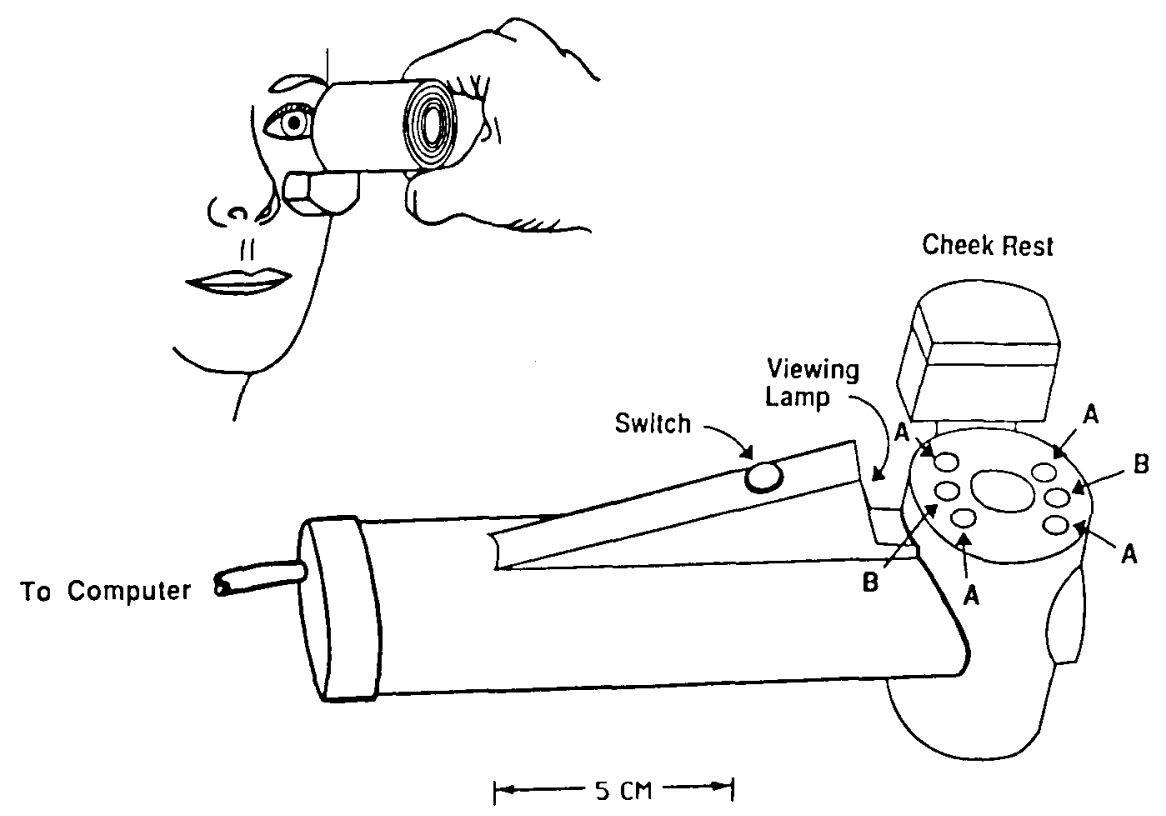

Figure 1. The infrared electronic pupilometer optical unit and its positioning on a subject's cheekbone. The 5-cm scale refers to the instrument schematic. $A$ and $B$ indicate location of infrared and high-intensity green stimulus pulse diodes, respectively.

ery $0.10 \mathrm{sec}$. Models currently available are also capable of measures every $0.05 \mathrm{sec}$. The image of the pupil from the reflected infrared light is stored in an $8 \mathrm{~K} \times 8 \mathrm{~K}$ static ram. The image sensor is a $256 \times 256$ rectangular pixel array. The computer analyzes this image to extract the pupil diameter in pixels and converts the measurement into millimeters. Measurement is made along the vertical axis of the pupil.

While the switch is depressed, an algorithmic circuit automatically adjusts the intensity of infrared illumination to an optimum level for conditions of ambient light, iris pigmentation, and pupil/iris contrast. The intensity of the infrared illumination at which the measurement is made is recorded, as IRED level, with the measurement data. The maximum infrared illumination intensity of the pupilometer is $7.5 \mathrm{~mW} / \mathrm{cm}^{2}$. Most measurements are made with infrared intensity levels of $0.75-1.5 \mathrm{~mW} / \mathrm{cm}^{2}$.

After the switch is released, the infrared diode intensity is decreased one IRED level, and two or three measures are made at $0.10-\mathrm{sec}$ intervals and compared with the last reading taken before the switch was released. If these values are within $0.1 \mathrm{~mm}$, the last value before the switch was released is entered as the initial diameter value. If the diameters differ by more than $0.1 \mathrm{~mm}$, "IRED level check failed" is displayed, and the aiming procedure and measurement cycle must be repeated. Improper aim, movement by operator or subject, blinking by the subject, or an inability of the computer to delineate the pupil/iris boundary can cause "IRED level check failed" messages.

When the switch is released, a pulse of light stimulates the pupil, and pupil diameters continue to be measured and stored every $0.10 \mathrm{sec}$. Three stimulus-pulse intensity- level settings are programmable: 1,2 , and 3 , corresponding to 9,18 , and $36 \mathrm{~lm} / \mathrm{ft}^{2}$. Duration of the stimulus pulse is programmable in 0.10 -sec increments. The stimulus pulse is a 2-mm diameter beam of light from a pair of high-intensity green diodes directed at the center of the pupil. The viewing lamp and the stimulus-pulse sources are positioned so that eyelashes do not interfere with the measurement.

The pupilometer scans the pupil for 2 or $3 \mathrm{sec}$ after the operating switch is released. Initial, minimum, and final pupil diameters are displayed in millimeters, along with the time of measurement and the elapsed time from the start of the stimulus pulse to the minimum diameter. At the end of the measurement cycle, a graph of the pupil diameters as a function of time appears on the host screen. Inspection of the graph confirms capture of valid pupil light-reflex data or allows the deletion of a measurement where a blink, a movement, or a misalignment caused artifacts. The operational sequence of the pupilometer components during a measurement cycle is illustrated in Figure 2.

The operating program presents menu bars to prompt the operator through the steps of identifying the subject and selecting the variable measurement parameters. Hard copies of pupil light-reflex plots and a summary of the digital data may be produced immediately after the measure is taken or later from the disk-stored data. Data can be stored in a format that allows later accumulation in a database. Three successive measurements are displayed simultaneously, to allow immediate comparison or detection of change with time.

Data can be manipulated or graphically displayed, using commercially available spreadsheet programs (e.g., 


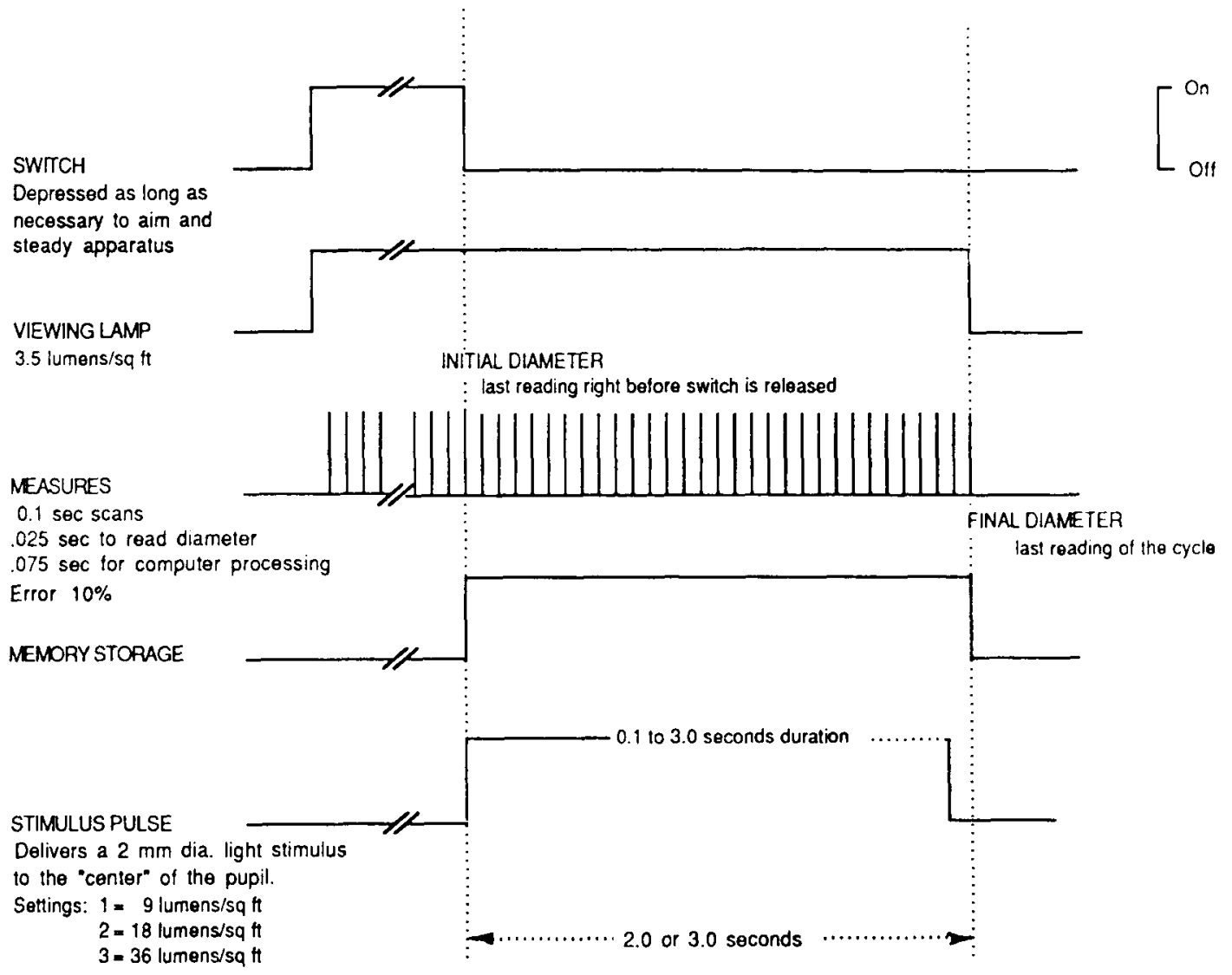

Figure 2. Operational sequence of each component of the pupilometer. Baseline is the "Off" condition; positive deflection from the baseline is the "On" condition.

Lotus 1-2-3) and a data management template supplied by the instrument vendor. Figure $\mathbf{3}$ shows the typical human light reflex as recorded by the pupilometer and the measurements that can be obtained from the data.

\section{STUDY 1}

\section{Operational Characteristics of the Pupilometer}

To evaluate the operational capabilities of the pupilometer over a range of potential pupil diameters, two pupil models were used. One consisted of a series of holes of different sizes drilled on $0.8-\mathrm{mm}$ sheet metal. The holes were drilled with $1.98,3.05,4.8,6.2,8.6$, and $10.3-\mathrm{mm}$ diameter drill bits. The other was a $4.5-\mathrm{mm}$ diameter model eye. The model eye was a hollow plastic hemisphere approximating the size of the human eyeball, in which a $4.5-\mathrm{mm}$ hole had been drilled through the center of a 12-mm diameter "iris" painted with brown enamel. The back of the model eye was painted flat black to approximate the iris/pupil contrast of the human eye.

An IBM-compatible computer, Leading Edge Model DR-1240, with a 12-in. monochrome display and a DC2011 disk drive, was used as the host computer. A Taxan Model KG-12NU-YN 12-in. monochrome monitor was used as an auxiliary monitor to provide immediate feed- back to the technician and thereby help to position the optical unit properly.

\section{Method}

Both the sheet-metal model and the pupilometer optical unit were clamped in a custom-fabricated stand. Diameter measurements of the holes were made at the focal distance of $28.6 \mathrm{~mm}$ (1.125 in.) specified by the manufacturer, with a stimulus-pulse level of $36 \mathrm{~lm} / \mathrm{ft}^{2}$. Six measures of each diameter were made, with stimulus-pulse durations of $0.5 \mathrm{sec}$. Six diameter measurements of each hole were also made with the CU camera at its instrument focal length. Measurements were made at $f / 22$, $1 / 15 \mathrm{sec}$, with a flash-intensity setting of 0 . The method for taking measurements was the same as that described below for human volunteers.

Measurements of the model eye were then made with the pupilometer and the model eye clamped in the stand at the instrument focal distance, and with positive and negative deviations at increments of $3.2 \mathrm{~mm}(.125 \mathrm{in}$.) from the instrument focal length. At each incremental distance, four measurements were made at a stimulus-pulse level of $36 \mathrm{~lm} / \mathrm{ft}^{2}$. All measurements were made with a stimulus-pulse duration of $0.5 \mathrm{sec}$. Measurements of the model eye pupil were also made with the $\mathrm{CU}$ camera at 


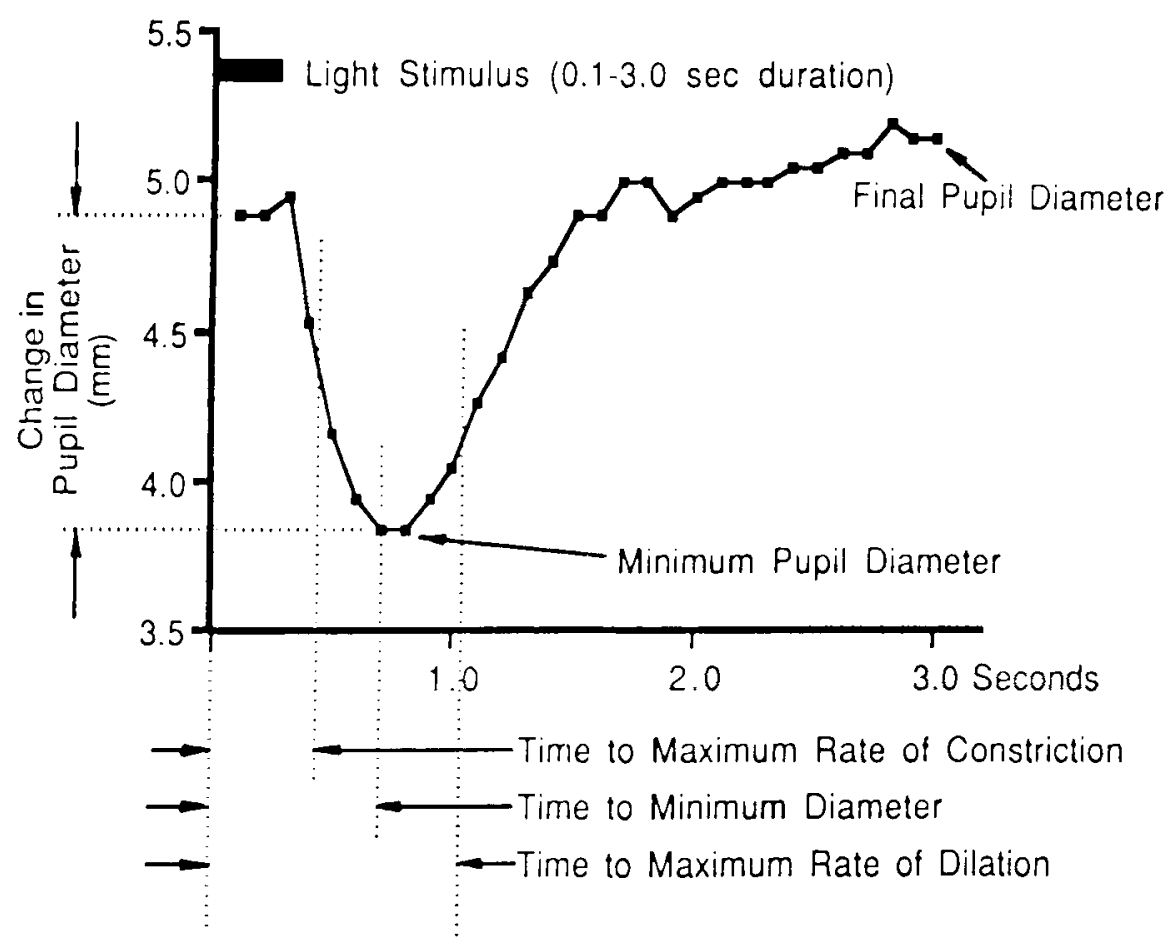

Figure 3. Typical pupillary light-reflex response curve from a human subject obtained with the pupilometer and the measurements that can be obtained from the data.

the same incremental deviations from its instrument focal length. Measurements were made at $\mathrm{f} / 22,1 / 15 \mathrm{sec}$, with a flash-intensity setting of 0 . Further measures of the model eye were also made at the instrument focal length with the stimulus-pulse level of $36 \mathrm{~lm} / \mathrm{ft}^{2}$ and with stimulus-pulse durations of $0.0,0.1,0.2,0.3,0.5,1.0$, 2.0 , and $3.0 \mathrm{sec}$.

Differences between the measured pupil size and $4.5 \mathrm{~mm}$ would assess measurement error due to deviations from instrument focal length. Because the model eye cannot produce a light reflex, the response curve recorded by the pupilometer should be a straight line at the 4.5 $\mathrm{mm}$ value. Deviations from the $4.5-\mathrm{mm}$ value would assess the consistency of the pupilometer electronics. Furthermore, deviations from the straight line as the stimuluspulse duration was increased would indicate that light from sources other than the instrument infrared are admitted as data signals.

\section{Results}

A Pearson correlation of 0.99 was obtained between pupilometer and CU camera measures of holes drilled in sheet metal (see Figure 4, panel A). Measurements of the model eye pupil at varying focal distances indicated that accurate measurements were assured only within about $3 \mathrm{~mm}$ of the pupilometer's focal length. A deviation of $\pm 3.2 \mathrm{~mm}(.125 \mathrm{in}$.) from the focal length produced measures that differed up to $34 \%$ from the actual $4.5-\mathrm{mm}$ value; a deviation of $\pm 6.4 \mathrm{~mm}$ (.25 in.) produced measures that differed up to $38 \%$ from the actual $4.5-\mathrm{mm}$ value. With the $\mathrm{CU}$ camera, a deviation of $\pm 6.4 \mathrm{~mm}$
(.25 in.) from its focal length produced measures that differed by less than $10 \%$ from the actual $4.5-\mathrm{mm}$ value. Figure 4, panel B, shows the diameters recorded as distance between each instrument and the $4.5-\mathrm{mm}$ model eye pupil was varied from each instrument's focal length.

At various focal distances, although the diameters of the model eye pupil recorded by the pupilometer varied from the actual diameter, the individual points of the model eye flat light-reflex curve varied no more than $0.2 \mathrm{~mm}$ from the initial diameter value recorded.

Measures of the model eye pupil at the pupilometer focal length and at stimulus-pulse level of $36 \mathrm{~lm} / \mathrm{ft}^{2}$ produced initial diameter measures that varied by up to $0.1 \mathrm{~mm}$ from the actual $4.5-\mathrm{mm}$ diameter of the model pupil. Varying the stimulus-pulse duration had no effect on measurements of the model eye pupil diameter.

\section{STUDY 2}

\section{Comparison of Data Collected \\ Using the Pupilometer and the CU Camera on Human Volunteers}

\section{Method}

During ongoing studies of the pharmacokinetics of morphine-related drugs (opioids) at the Addiction Research Center, pupil-diameter measurements were made concurrently with both the pupilometer and the CU camera. Six male volunteers with histories of opioid abuse participated while residing in the Residential Research Unit of the Addiction Research Center. Each was given a placebo and a number of doses of an opioid. The volun- 

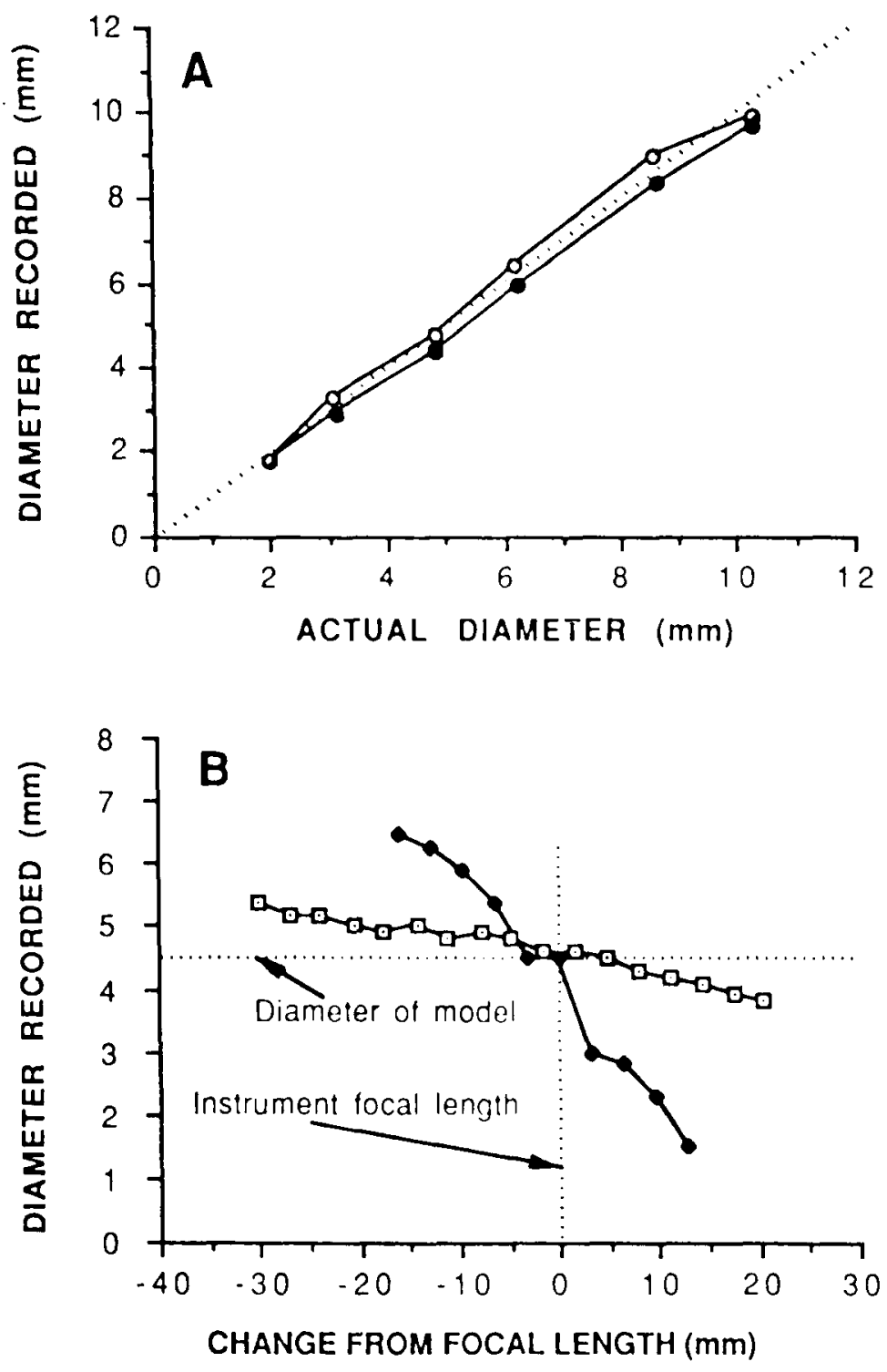

Figure 4. Panel A: Measurements by pupilometer and CU camera of 2- to $10-\mathrm{mm}$ diameter holes in sheet metal. Open circles are pupilometer measures and filled circles are CU camera measures. Panel B: Measurements of a 4.5-mm diameter model eye pupil with the pupilometer and CU camera, as the distance between the instruments and the model eye was varied from the focal length of each instrument. The filled diamonds are pupilometer measures. The open squares are $\mathrm{CU}$ camera measures.

teers had been medically screened and had provided informed consent to participate in the study. The mean ages and body weights of the subjects were 34.8 years $(S D=$ $2.8)$ and $74.4 \mathrm{~kg}(S D=13.3)$. Additional procedural details and results of the opioid study will be published elsewhere (E. J. Cone, personal communication).

The CU camera measurements of the pupil, using a flash unit and a ratio multiplier, produced a magnified $(2 \times)$ photograph of the pupil. A millimeter scale was made an integral part of the manufacturer-supplied lensto-subject spacer. Pupil diameters were then determined, measuring the image and comparing the value to the scale photographed with the pupil. Pupil diameters from the $\mathrm{CU}$ camera were obtained by measuring along the horizontal axis of the pupil. As an internal check, the same researcher compared measurements along the vertical and the horizontal axes of the photographed pupil of 1 subject. As a further check, results were compared from measurements along the horizontal axis of the pupil made by two researchers using the same $\mathrm{CU}$ photographic data from another subject.

Measurements with the two systems were made approximately 1 min apart. Pupilometer measurements were taken prior to the CU camera measurements, since the photoflash of the CU camera was assumed to produce a longer lasting alteration of pupil function than the light 

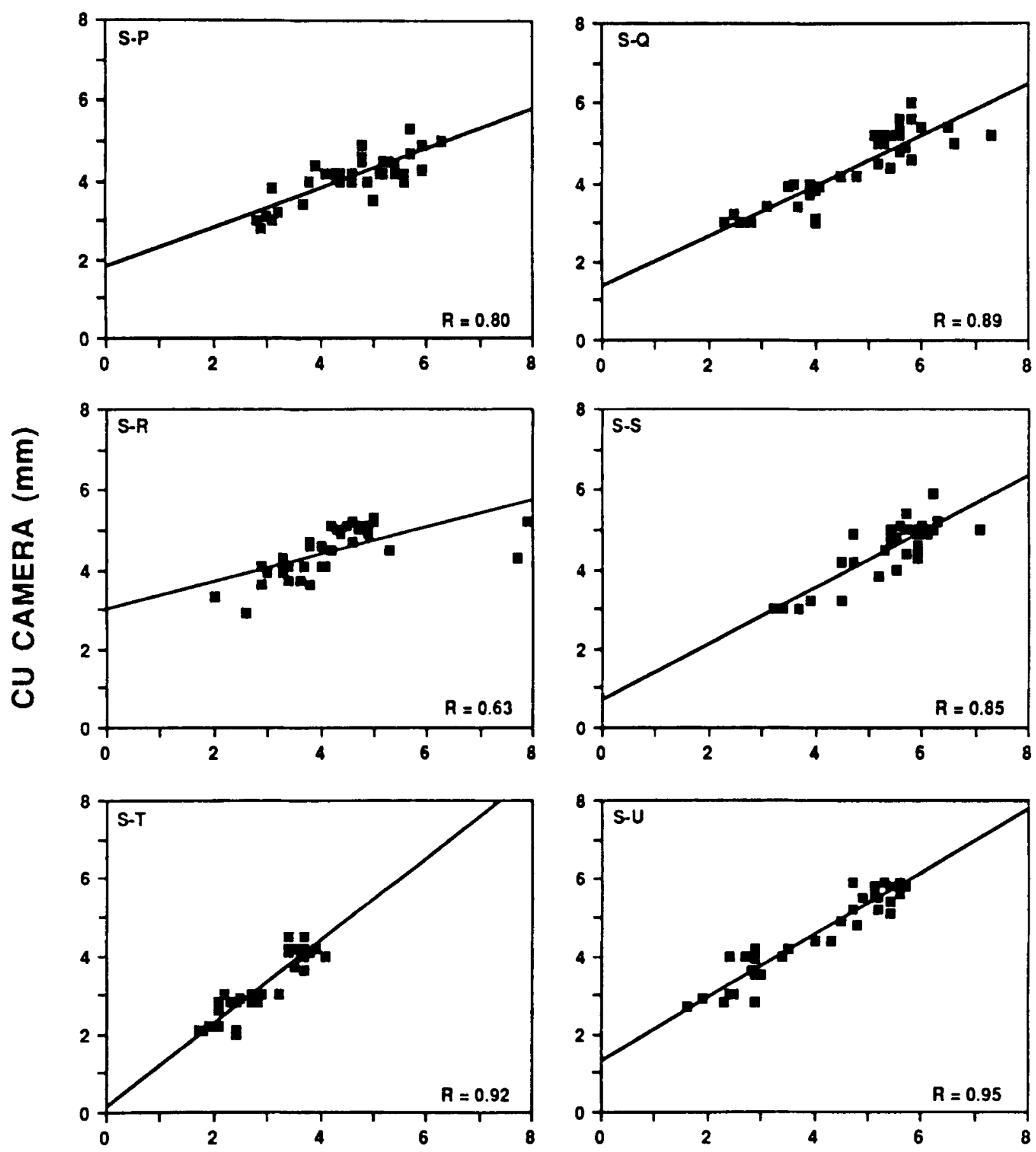

\section{PUPILOMETER $(\mathrm{mm})$}

Figure 5. Correlations between pupil diameters measured along the vertical axis of the pupil with the pupilometer and measurements along the horizontal axis with the CU camera from 6 subjects.

stimulus of the pupilometer would. For comparison of the static pupillary data from the two systems, the data from the CU photograph were compared with the initial diameter recorded by the pupilometer. With 4 subjects, after the light reflex was measured with the usual stimuluspulse duration of $0.5 \mathrm{sec}$, two more measurements were made about $1 \mathrm{~min}$ apart at stimulus-pulse durations of 1.0 and $2.0 \mathrm{sec}$.

\section{Results}

Across subjects, the Pearson correlations between the initial diameters recorded by the pupilometer and by the 


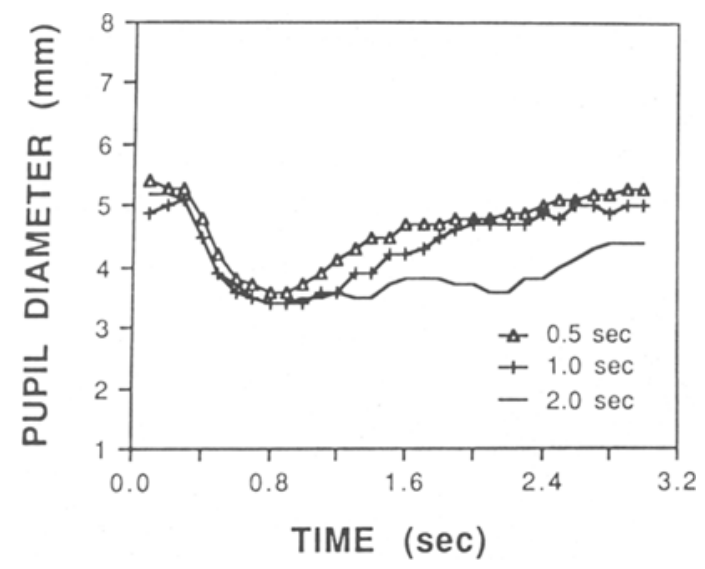

Figure 6. Data from a male subject were selected as a representative illustration of the effect on light reflex after increasing only the stimulus-pulse duration from 0.5 to 1.0 and $2.0 \mathrm{sec}$. The time between measurements is about $1 \mathrm{~min}$. The stimulus-lamp intensity was $9 \mathrm{Im} / \mathrm{ft}^{2}$.

CU camera ranged between 0.63 and 0.95 (see Figure 5). The correlation coefficient between measures from the vertical and horizontal axes of the pupil as measured from the CU camera photographs was 0.97 , indicating that the results were virtually identical whether measurements were based on the vertical or the horizontal axis. The correlation coefficient between measures made from the $\mathrm{CU}$ photographic record along the horizontal pupil diameters by two technicians was 0.98 .

As the stimulus-pulse duration was increased from 0.5 to 1.0 and $2.0 \mathrm{sec}$, parameters of the light reflex were unaffected up to about $1.2 \mathrm{sec}$. For example, the latency was $0.2-0.3 \mathrm{sec}$, and maximum constriction was seen 0.8-1.0 sec after the stimulus pulse was initiated. The redilation curve after stimulus-pulse durations of 0.5 and $1.0 \mathrm{sec}$ seemed not to differ. With stimulus-pulse durations of $2.0 \mathrm{sec}$, however, the pupil seemed to begin redilating from 1.0 to $1.2 \mathrm{sec}$, then reconstricted for the remaining 1.0-0.8 sec of the stimulus-pulse duration (see Figure 6).

Preliminary data indicate that the light reflex may reveal opioid-induced changes in pupillary dynamics. For example, Figure 7 shows dose-related changes in pupil diameter and light reflex after two doses of heroin.

\section{Discussion}

Under bench-top conditions, the pupilometer compares very well with the CU camera when diameters between 2 and $10 \mathrm{~mm}$ are measured. It appears that the pupilometer does not admit as data signals light other than infrared. Under normal conditions, this infrared light would originate from the pupilometer, and ambient light conditions should not affect the accuracy of measurements. However, when deviations in distance between the pupil and instrument are increased, measurement error increases at a faster rate with the pupilometer than with the $\mathrm{CU}$ camera.

Somewhat more time is involved in training staff to use the pupilometer as opposed to the CU camera. Even with the pupilometer's cheekrest seated on the subject's cheekbone, in attempting to obtain the proper image of the pupil on the monitor, it is possible to rotate the pupilometer out of parallel to the pupil/iris plane and thus occasionally to take measures at focal distances that may significantly deviate from the focal length of the instrument.

In a clinical setting, static pupil-diameter measurements made with the pupilometer compare well with the photographic measurements made with the CU camera. The portability of the pupilometer, due to its ability to function as an accessory to a lap-top computer, coupled with its ability to measure pupil diameters rapidly, at 0.10 - and 0.05 -sec intervals, lends it to convenient quantitative anal-

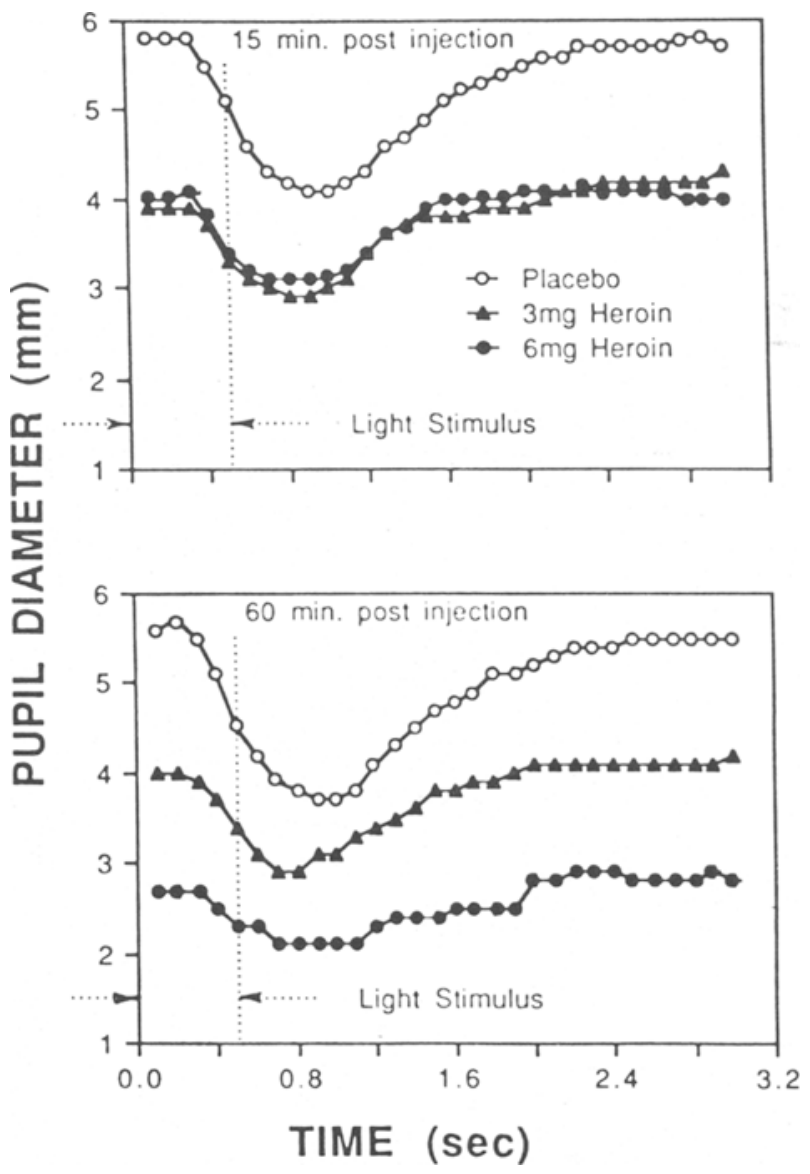

Figure 7. Data from a male subject were selected as a representative illustration of the effects of heroin on the light reflex. The stimulus-pulse duration was $0.5 \mathrm{sec}$ and the stimulus-lamp intensity was $9 \mathrm{~lm} / \mathrm{ft}^{2}$. The upper panel presents data recorded $15 \mathrm{~min}$ after the heroin injection, and the lower panel presents data recorded 60 min after the heroin injection. 
yses of the static and dynamic effects of drugs on the pupil. The ability of the pupilometer to record the light reflex may provide an additional means of quantitatively measuring drug action. Subsequent studies are being done in our laboratory to investigate the degree to which such pupillary effects are specific to drug classes.

\section{REFERENCES}

Henningfield, J. E., Miyasato, K., Johnson, R. E., \& JasinSKI, D. R. (1983). Rapid physiologic effects of nicotine in humans and selective blockade of behavioral effects by mecamylamine. In $\mathbf{L}$. S. Harris (Ed.), Problems of drug dependence, 1982: Proceedings of the 44th Annual Scientific Meeting. The Committee on Problems of Drug Dependence, Inc. (National Institute on Drug Abuse Research Monograph 43, pp. 259-265). Washington, DC: U.S. Government Printing Office.

HimmelsBaCH, C. K. (1941). The morphine abstinence syndrome, its nature and treatment. Annals of Internal Medicine, 15, 829-839.

JASINSKI, D. R., \& MARTIN, W. R. (1967). Evaluation of a new photo- graphic method for assessing pupil diameters. Clinical Pharmacology \& Therapeutics, 8, 271-272.

Marquardt, W. G., Martin, W. R., Jasinski, D. R. (1967). The use of the Polaroid CU camera in pupillography. International Journal of the Addictions, 2, 301-304.

Pickworth, W. B., \& LeE, H. (1988). Pupil size and the light reflex over a course of buprenorphine administration. Pharmacologist, 30 , A196.

SMITh, S. A., \& DEwhIRst, R. R. (1986). A simple diagnostic test for pupillary abnormality in diabetic autonomic neuropathy. Diabetic Medicine, 3, 38-41.

Tennant, F. (1988). The rapid eye test to detect drug abuse. Postgraduate Medicine, 84, 108-114.

\section{NOTE}

1. The Fairville Medical Optics Pupilscan is priced at $\$ 6,500$ with software included. The supplier is located at 1222 Norbee Drive, Wilmington, DE 18903.

(Manuscript received May 24, 1989; revision accepted for publication August 24, 1989.) 